\title{
PTEN loss represses glioblastoma tumor initiating cell differentiation via inactivation of Lgl1
}

\author{
Alexander Gont ${ }^{1,2}$, Jennifer E L Hanson ${ }^{1}$, Sylvie J Lavictoire ${ }^{1}$, Doris A E Parolin ${ }^{1}$ \\ Manijeh Daneshmand ${ }^{1,4}$, Ian J Restall ${ }^{1,2}$, Mathieu Soucie ${ }^{1,3}$, Garth Nicholas ${ }^{1,5}$, John \\ Woulfe $^{1,2,4}$, Amin Kassam ${ }^{5}$, Vasco F Da Silva ${ }^{5}$ and Ian AJ Lorimer ${ }^{1,2,6}$ \\ ${ }^{1}$ Centre for Cancer Therapeutics, Ottawa Hospital Research Institute, 501 Smyth Road, Ottawa, K1H 8L6, Canada \\ 2 Department of Biochemistry, Microbiology and Immunology, University of Ottawa, Ottawa, Ontario, Canada \\ ${ }^{3}$ Faculty of Science, University of Ottawa, Ottawa, Ontario, Canada \\ ${ }^{4}$ Department of Pathology and Laboratory Medicine, University of Ottawa, Ottawa, Ontario, Canada \\ ${ }^{5}$ Department of Surgery, University of Ottawa, Ottawa, Ontario, Canada \\ ${ }^{6}$ Department of Medicine, University of Ottawa, Ottawa, Ontario, Canada
}

Correspondence to: Ian A. J. Lorimer, email: ilorimer@ohri.ca

Keywords: glioblastoma, glioma, PTEN, PKCI, Lgl, tumor initiating cell

Received: July 8, $2013 \quad$ Accepted: July 19, $2013 \quad$ Published: July 21, 2013

This is an open-access article distributed under the terms of the Creative Commons Attribution License, which permits unrestricted use, distribution, and reproduction in any medium, provided the original author and source are credited.

ABSTRACT:

Glioblastoma multiforme is an aggressive and incurable type of brain tumor. A subset of undifferentiated glioblastoma cells, known as glioblastoma tumor initiating cells (GTICs), has an essential role in the malignancy of this disease and also appears to mediate resistance to radiation therapy and chemotherapy. GTICs retain the ability to differentiate into cells with reduced malignant potential, but the signaling pathways controlling differentiation are not fully understood at this time. PTEN IOSS is a very common in glioblastoma multiforme and leads to aberrant activation of the phosphoinositide 3-kinase pathway. Increased signalling through this pathway leads to activation of multiple protein kinases, including atypical protein kinase $\mathbf{C}$. In Drosophila, active atypical protein kinase $\mathbf{C}$ has been shown to promote the selfrenewal of neuroblasts, inhibiting their differentiation along a neuronal lineage. This effect is mediated by atypical protein kinase c-mediated phosphorylation and inactivation of Lgl, a protein that was first characterized as a tumour suppressor in Drosophila. The effects of the atypical protein kinase C/Lgl pathway on the differentiation status of GTICs, and its potential link to PTEN loss, have not been assessed previously. Here we show that PTEN loss leads to the phosphorylation and inactivation of $\mathbf{L g l}$ by atypical protein kinase $\mathrm{C}$ in glioblastoma cells. Re-expression of PTEN in GTICs promoted their differentiation along a neuronal lineage. This effect was also seen when atypical protein kinase $\mathbf{C}$ was knocked down using RNA interference, and when a non-phosphorylatable, constitutively active form of Lgl was expressed in GTICs. Thus PTEN loss, acting via atypical protein kinase C activation and Lgl inactivation, helps to maintain GTICs in an undifferentiated state.

\section{INTRODUCTION}

Glioblastoma multiforme is an aggressive type of adult brain tumor. Surgery is not curative, as this disease invariably exhibits extensive intracerebral dissemination at the time of diagnosis. Patients are also commonly treated with radiation and the alkylating agent temozolomide; this prolongs survival but is also not curative [1]. A characteristic histological feature of glioblastoma is that glioblastoma cells show considerable phenotypic heterogeneity. One factor contributing to this heterogeneity is that glioblastoma cells exist in different differentiation states [2]. A subset of glioblastoma cells are relatively undifferentiated; these are variously referred 
to as glioblastoma stem cells, glioblastoma stem-like cells or glioblastoma tumor-initiating cells $[3,4]$. The latter term is based on one of their defining properties, which is the ability to regenerate a tumor resembling the patient's original tumor when implanted in the brain of an immunocompromised mouse [3]. These cells share some properties with normal adult neural stem cells, including the expression of stem cell-associated genes and the ability to differentiate along multiple lineages [5]. Current data suggests that these undifferentiated cells are resistant to radiation therapy and temozolomide and therefore play a key role in glioblastoma recurrence $[6,7]$.

The genetics of glioblastoma are now understood in some detail. Loss of PTEN is a very frequent event in glioblastoma, with hemizygous or homozygous deletions occurring in over $90 \%$ of primary glioblastomas [8]. PTEN catalyzes the inactivation of the second messenger phosphatidylinositol 3,4,5 trisphosphate. This second messenger is produced by the enzyme phosphoinositide 3' kinase (PI 3-kinase) after activation by tyrosine kinase receptors. Loss of PTEN therefore results in increased signaling through the PI 3-kinase pathway. Other glioblastoma mutations, including mutations in the $E G F R$, $P I K 3 C A$ and $P I K 3 R 1$ genes, also activate this pathway $[8,9]$. While much attention has focused on the role of $\mathrm{Akt} / \mathrm{PKB}$ as a downstream mediator in the PI 3-kinase pathway, PI 3-kinase signaling results in the activation of multiple other downstream kinases [10]. This includes atypical protein kinase $\mathrm{C}$ (PKC) family members [11]. There are two atypical PKCs in humans, $\mathrm{PKC} \zeta$ and $\mathrm{PKC}$. Of these, $\mathrm{PKCl}$ is the most ubiquitously expressed in tissues and overexpressed $\mathrm{PKCl}$ has been shown to have the properties of an oncogene in several different tumor types [12]. In studies using human glioblastoma cell lines, $\mathrm{PKCl}$ has been shown to have a role in both proliferation and invasion $[13,14,15]$. Relatively little is known about the kinase substrates that mediate these effects. One of the more well-characterized substrates of the atypical PKCs is a protein known as Lgl.

Lethal Giant larvae (Lgl) was first identified as an allele in Drosophila that, when mutated, gave rise to a neoplastic phenotype characterized by overgrowth of imaginal epithelia and brain tissue [16]. In Drosophila brain tissue, this overgrowth is the result of neuroblasts preferentially undergoing self-renewal rather than differentiating into neurons [17]. Mammals have two genes with homology to Drosophila Lgl: LGL1, which in mice is broadly expressed with the highest expression in brain; and $L G L 2$, which shows a more restricted expression pattern [18]. Knockout of Lgl1 in mice causes a brain dysplasia phenotype [18]. At the cellular level, Lgl proteins have multiple functions related to cell polarity, including asymmetric cell division [19]. Additional cell polarity functions include the maintenance of apical/ basolateral cell polarity (in epithelial tissue); polarized exocytosis; and cell motility [20,21]. Lgl proteins bind non-muscle myosin II and associate with the inner leaflet of the plasma membrane. They also bind the scaffolding protein Par6. Atypical PKCs also bind to Par6 and in this complex they are able to phosphorylate and inactivate Lgl. Inactivated Lgl no longer associates with the plasma membrane or with non-muscle myosin II [22].

Given its original characterization as a tumor suppressor in Drosophila, a possible role for Lgl as a tumor suppressor in human cancers has also been investigated. Human Lgll can rescue Drosophila Lgl mutants, showing conservation of function [23]. Human Lgl1 mRNA and protein are reduced in multiple cancer types including colorectal cancer and melanoma [23,24,25]. This reduced expression is not due to either Lgl1 gene mutations or promoter methylation, but instead is due to transcriptional repression [26]. Although Lgl1 shows strong expression in brain and is known to control brain development in both Drosophila and mammals, there has been no detailed investigation of the role of Lgl1 in glioblastoma to date. Here we show that in glioblastoma, PTEN loss results in the inactivation of Lgll by phosphorylation. This inactivation of Lgl1 has a key function in the maintenance of undifferentiated glioblastoma tumor-initiating cell populations.

\section{RESULTS}

\section{Constitutive phosphorylation of Lgl1 in glioblastoma cells}

A lentiviral vector for constitutive expression of Lgl1 was constructed and used to express Lgl1 in U87MG human glioblastoma cells. In addition a second lentiviral vector was made to express a non-phosphorylatable, constitutively active Lg11 (designated Lg13SA), in which the three major Lgl1 phosphorylation sites identified by Yamanaka et al. were mutated to alanine [27]. Transduced Lg11 and Lg13SA were expressed at similar levels in U87MG glioblastoma cells (Figure 1A). U87MG cells express low levels of endogenous Lg11, visible as a faint band in Figure 1A in the blot probed with Lgl1 antibody. To detect phosphorylated transduced Lg11, a phospho(Ser) PKC substrate antibody was used; in total cell extracts this labeled a prominent band of the expected size in cells transduced with Lgl1, but not in cells transduced with Lg13SA (Figure 1B). Knockdown of Lgl1 with two different Lgl1 RNA duplexes also decreased the intensity of the band detected with the phospho-(Ser) PKC substrate antibody, confirming its identity (Figure 1C). Knockdown of $\mathrm{PKCl}$ (the sole atypical $\mathrm{PKC}$ isoform expressed in U87MG cells [14]) also reduced the intensity of this band, confirming that $\mathrm{PKC}$ is responsible for $\mathrm{Lgl1}$ phosphorylation in these cells (Figure 1D). 


\section{Effects of PTEN on Lgl1 activation}

To assess the effects of PTEN on Lgl1 phosphorylation, a doxycycline-inducible expression system for PTEN was generated in U87MG cells, which do not express PTEN due to a mutation [28]. This gave rapid, inducible expression of PTEN that was active, as assessed by its ability to decrease phosphorylation of Akt and $\mathrm{PKCl}$ (Figure 2A). These cells were transduced with Lgl1 cDNA and Lgl1 phosphorylation was assessed with phospho-(Ser) PKC substrate antibody as above. Induction of PTEN decreased levels of phosphorylated Lgl1 without significantly affecting the total levels of Lgl1 protein (Figure 2B). Lgl1 in its active form is membranelocalized and inactivation by phosphorylation causes its translocation to the cytosol. In U87MG cells, transduced wild-type Lgl1 was localized in the cytoplasm. Induction of PTEN altered the subcellular localization of wild- type Lgl1, such that it was predominantly membraneassociated, consistent with its restoration to an active form (Figure 2C).

\section{Membrane association of a non-phosphorylatable Lgl1 mutant in glioblastoma cells}

A doxycycline-inducible expression system to express either wild-type Lgl or Lgl3SA was also made in U87MG cells. As with the constitutive expression system, both proteins were expressed at similar levels and were detectable within $6 \mathrm{~h}$ after induction of expression with doxycycline (Figure 3A). Induced Lgl and Lgl3SA showed different subcellular localizations in glioblastoma cells, with Lgl being predominantly cytoplasmic and Lgl3SA showing significant membrane association (Figure 3B). This membrane localization was particularly pronounced in mitotic cells; double staining for both Lgl and non-
A.

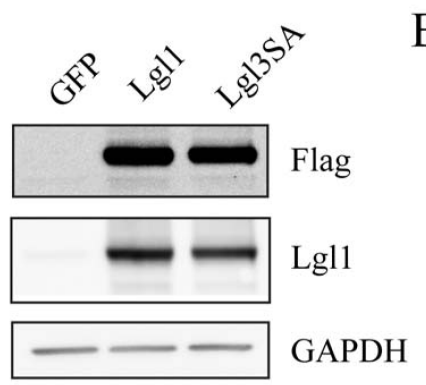

C.

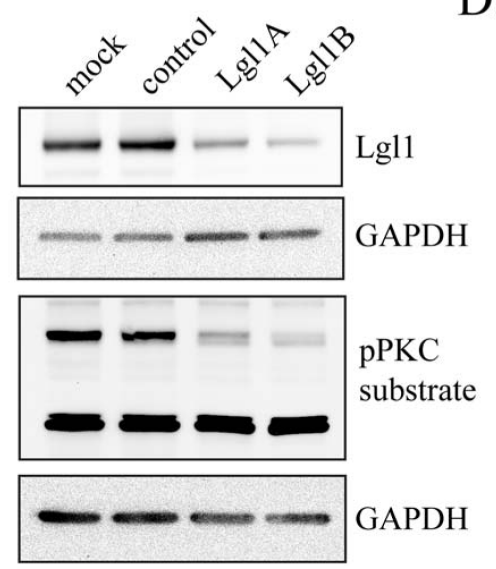

B.

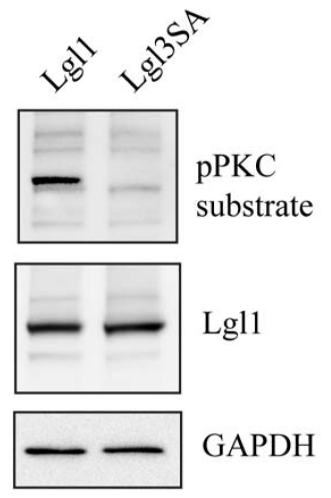

D.

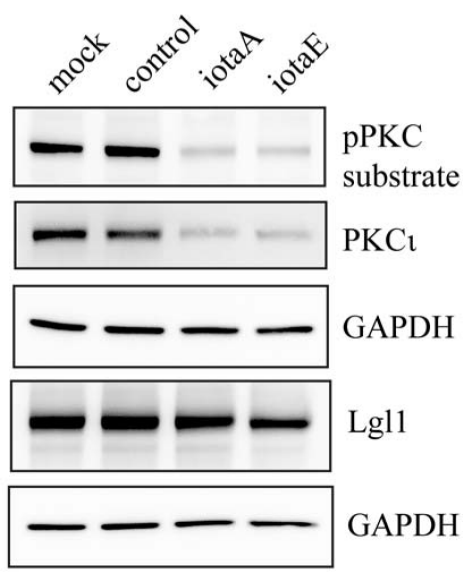

Figure 1: Lgl1 is constitutively phosphorylated by PKCı in PTEN-null U87MG cells. A. U87MG cells were transduced with lentiviral vectors expressing GFP (as a control), wild-type Lgl1, or a mutant non-phosphorylatable Lgl1 (Lgl3SA). Expression of Lgl1 was assessed by Western blotting of total cell lysates using antibodies to Flag epitope, Lgl1 and GAPDH (as a loading control); B. U87MG cells were transduced with lentiviral vectors expressing either wild-type Lgl1 or Lgl3SA. Total cell lysates were analyzed by Western blotting using antibodies to phospho-PKC substrate, Lg11 and GAPDH; C. U87MG cell transduced with lentiviral vector expressing wild-type Lg11 were mock transfected, transfected with control RNA duplex, or transfected with two different RNA duplexes targeting Lgl1 (LglA and LglB). $48 \mathrm{~h}$ after transfection, total cell lysates were collected and analyzed on separate Western blots with antibodies to Lglland phosphoPKC substrate (GAPDH loading controls are shown for each blot). D. U87MG cell transduced with lentiviral vector expressing wild-type Lgl1 were mock transfected, transfected with control RNA duplex, or transfected with two different RNA duplexes targeting PKCl (iotaA and iotaE). $48 \mathrm{~h}$ after transfection, total cell lysates were collected and analyzed by Western blotting for phospho-PKC substrate, $\mathrm{PKC}$ and Lgl1. 
muscle myosin II (a known Lgl binding partner) in mitotic cells showed that Lgl3SA, but not Lgl, colocalized with non-muscle myosin II.

\section{Characterization of glioblastoma tumor initiating cells (GTICs)}

GTICs were isolated from patients undergoing surgery for glioblastoma at The Ottawa Hospital. Cells were isolated following the procedure described by
Pollard et al., in which GTICs are plated directly onto laminin-coated plates [5]. Cells were cultured in 5\% $\mathrm{O}_{2}$, which significantly enhanced their growth rate compared to growth in atmospheric oxygen levels (20\%). Enhanced growth in $5 \% \mathrm{O}_{2}$ has been observed previously for neural stem cells [29] and may be due in part to reduced senescence [30]. This level is also more physiologically appropriate than atmospheric oxygen levels $(20 \%)$, as it is similar to oxygen levels in the adult brain [29]. GTICs were used at low passage numbers (less than 20 passages),

A.
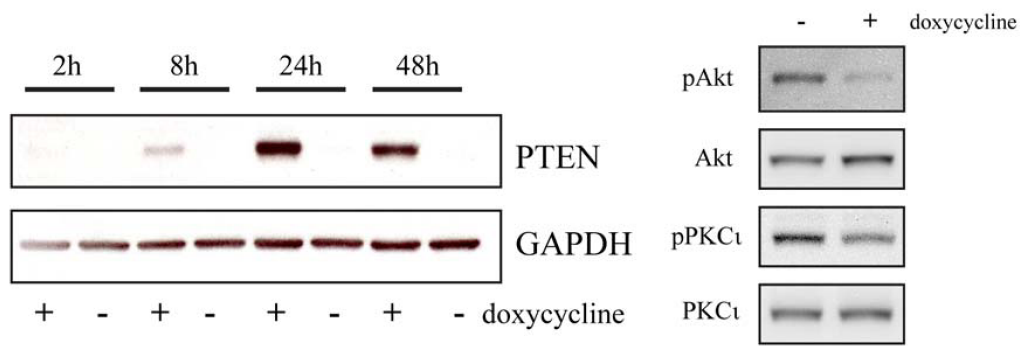

B.
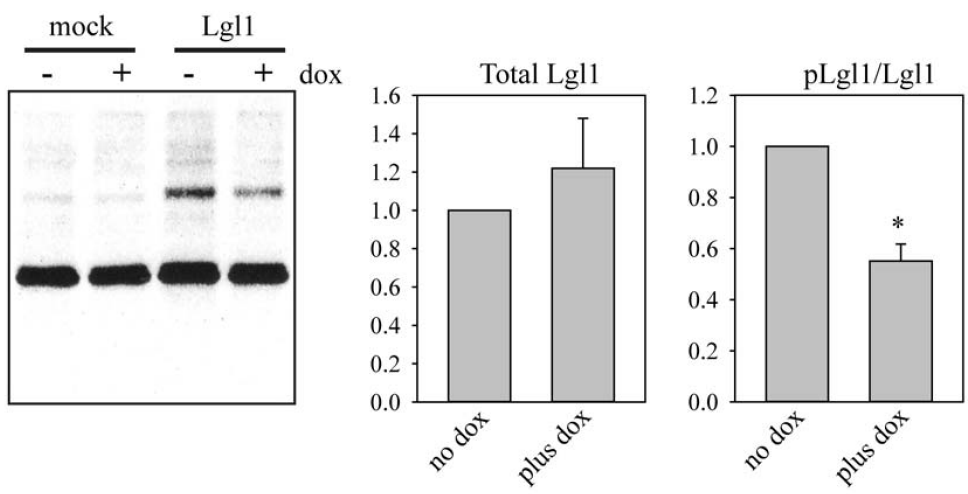

C.
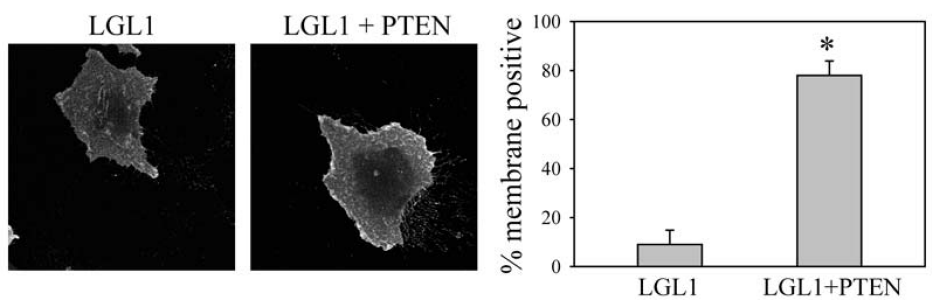

Figure 2: Restoration of PTEN expression reduces Lgl1 phosphorylation and promotes its membrane association. A. U87MG cells were transduced with lentiviral vectors expressing Tet activator and Tet-inducible PTEN. Cells were treated with or without $100 \mathrm{ng} / \mathrm{ml}$ doxycycline for the indicated period of time. Total cell lysates were then analyzed by Western blotting for PTEN expression (left panels). Total cell lysates from cell treated for $48 \mathrm{~h}$ with $100 \mathrm{ng} / \mathrm{ml}$ doxycycline were also analyzed by Western blotting for total and phosphorylated Akt and $\mathrm{PKCl}$ (right panels). B. U87MG cells with doxycycline-inducible PTEN were treated for two days with doxycycline and then either mock-transduced or transduced with lentiviral vector constitutively expressing wild-type Lg11. Total cell lysates were collected two days after transduction and analyzed by Western blotting with phospho-PKC substrate antibody (shown), Lgl1 antibody and GAPDH antibody (not shown). Data from three independent experiments were analyzed for levels of total and phosphorylated Lgl1 (bar graphs at left). Data were first normalized to GAPDH levels and are shown normalized to the no doxycycline condition in the bar graphs. Data are shown as the mean \pm SD. $*$ indicates a p value less than 0.05 . C. U87MG cells with doxycycline-inducible PTEN were treated for two days with doxycycline and then transduced with lentiviral vector expressing wild-type Lgl1. Two days after transduction, cells were fixed and analyzed by immunofluorescence with anti-Flag antibody. Examples of immunofluorescence staining are shown in the left two panels. The right panel shows the analysis for membrane localization from 15 randomly selected images per experiment assessed by an observer (IR) blinded to the treatment conditions. Data are the mean SD from three separate experiments. * indicates a p value less than 0.05 . 
as this has been shown to be important for maintaining multipotency [31]. Analysis of GTIC cultures from three different patients showed that they all expressed Lgl1 and $\mathrm{PKCl}$, with expression of Lgll being considerably higher than in U87MG cells (Figure 4A). Cells from two patients showed detectable expression of PTEN, while cells from one patient (PriGO8A cells) showed a complete absence of PTEN expression, as in U87MG cells (Figure 4A). The latter were chosen for further detailed analysis. Chromogenic in situ hybridization showed that PriGO8A cells had three copies of the EGFR gene, likely reflecting a gain of chromosome 7 , a characteristic genetic feature of glioblastoma (Figure 4B). When grown in the absence of laminin, the cells readily formed neurospheres resembling those seen in neural stem cell culture (Figure 4C). The cells also uniformly stained positive for nestin, a standard marker of neural stem cells (Figure 4D). When injected intracerebrally into immunocompromised mice, these cells formed a diffuse glioblastoma that was highly invasive (Figure 5A). The pattern of invasion was typical of glioblastoma, with extensive movement of cells into the uninjected hemisphere occurring along the corpus callosum. Thus these cells have the characteristic features of GTICs described in previous publications [5,32].

The ability of PriGO8A cells to differentiate in response to standard differentiation induction methods (serum addition, with or without growth factor withdrawal) was assessed (Figure $5 \mathrm{~B}$ and $\mathrm{C}$ ). To assess neuronal differentiation, neuron-specific class III $\beta$-tubulin (TUJ1) antibody was used; differentiation along the astrocytic lineage was assessed using antibody to glial fibrillary acidic protein (GFAP). These markers have been used extensively to assess differentiation along neuronal and astrocytic lineages in both GTICs and normal adult neural stem cells [3].The addition of serum, either in the presence or absence of growth factors, increased the

A.
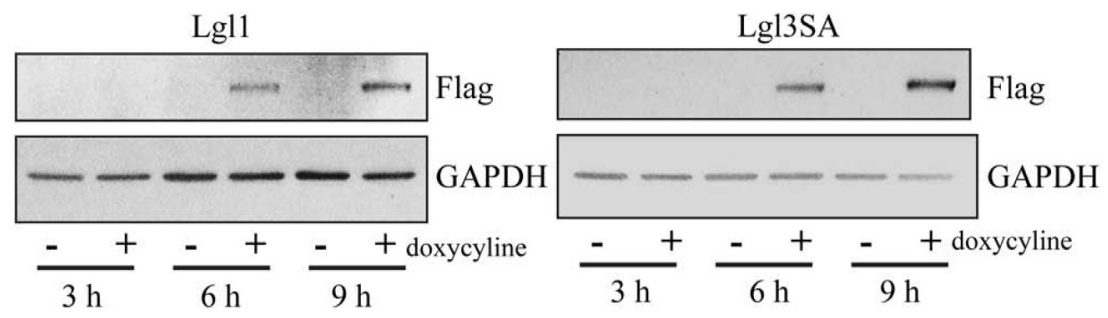

B.
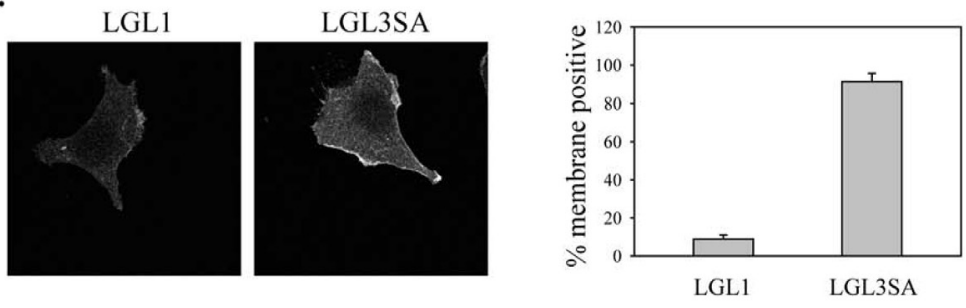

C.

DAPI
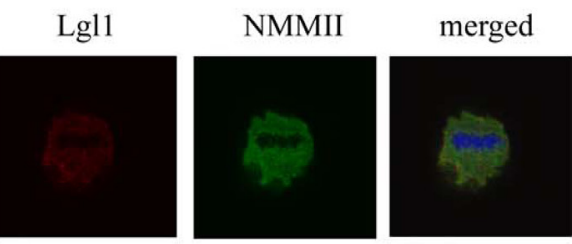

$\operatorname{Lgl1}(+$ dox $)$
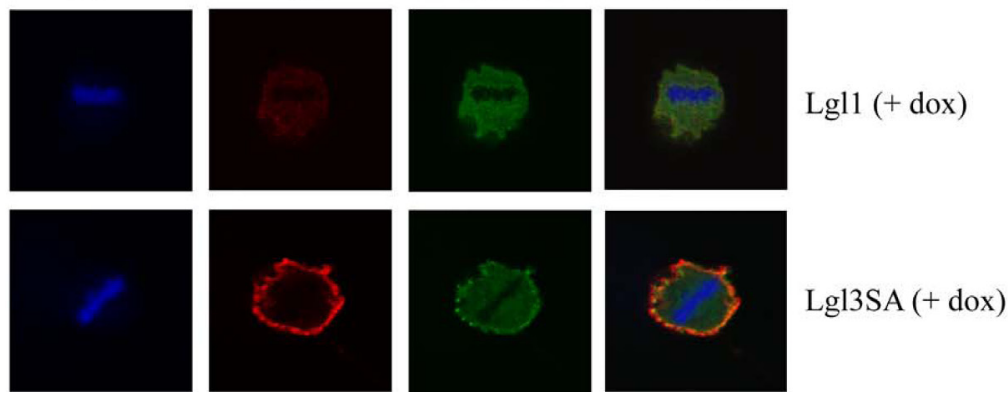

Figure 3: Non-phosphorylatable Lgl1 constitutively associates with the cell membrane in U87MG cells. U87MG cells were transduced with lentiviral vectors expressing tet activator and tet-inducible $\mathrm{Lgl}$ or Lgl3SA. Cells were treated with $100 \mathrm{ng} / \mathrm{ml}$ doxycycline for the indicated periods of time and then analyzed by Western blotting with anti-Flag antibody. B. U87MG cells with inducible Lgl1 or Lgl3SA expression were treated with $100 \mathrm{ng} / \mathrm{ml}$ doxycycline for 4 days and then analyzed by immunofluorescence microscopy using antiFlag antibody. Membrane localization was quantitated as describe in Figure 2C. C. U87MG cells with inducible Lgl1 or Lgl3SA expression were treated with $100 \mathrm{ng} / \mathrm{ml}$ doxycycline for 4 days and then analyzed by confocal immunofluorescence microscopy with antibodies to Flag epitope (red) and non-muscle myosin IIa (green). Cells were counterstained with DAPI. Examples of confocal images of mitotic cells expressing Lg11 and Lg13SA are shown. 


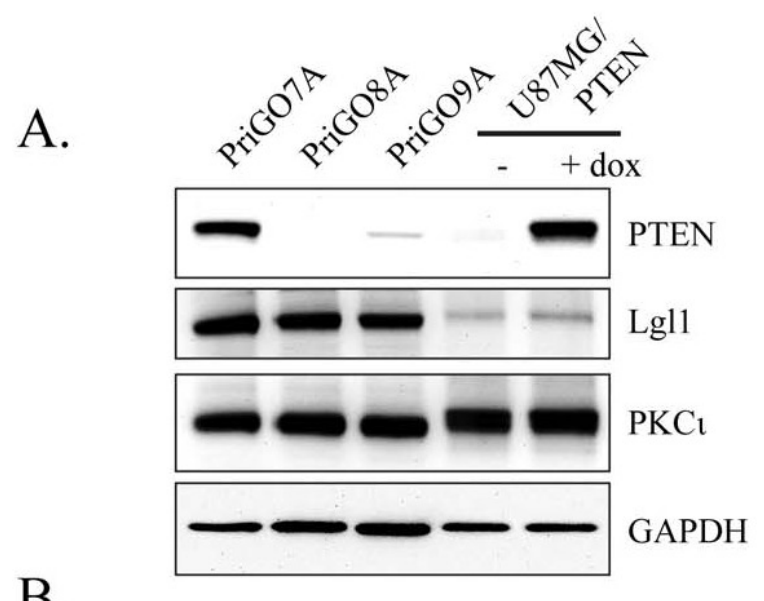

B.
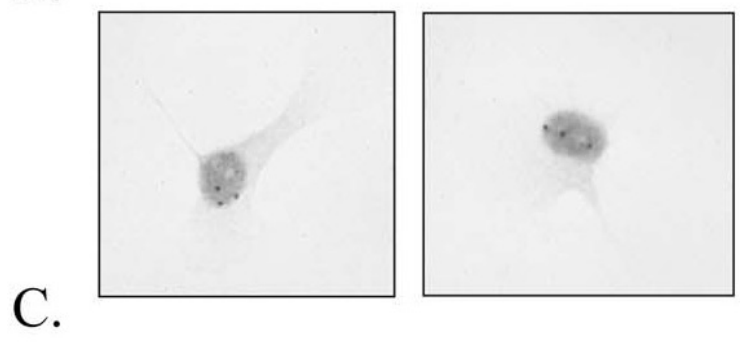

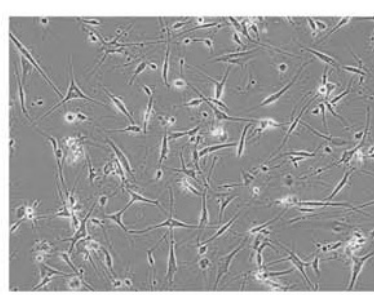

laminin D.

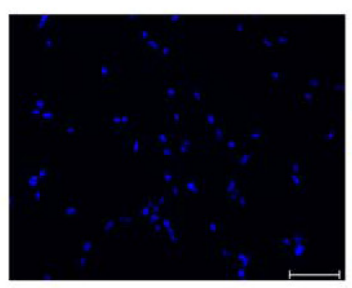

DAPI no primary

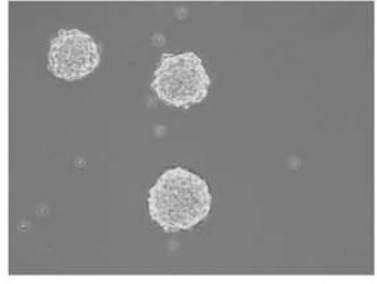

no laminin

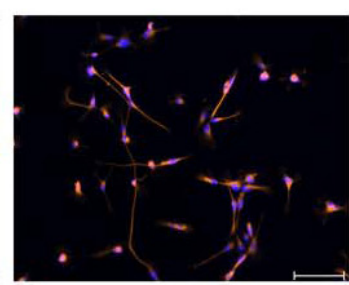

DAPI

nestin
Figure 4: Characterization of PriGO8A cells. A. Western blot analysis of PTEN, Lgll and $\mathrm{PKCr}$ expression in GTIC cultures from three patients. Total cell extracts from U87MG cells with doxycycline-inducible PTEN were also run for comparison. B. Chromogenic in situ hybridization was performed on PriGO8A cells using an EGFR probe. Examples of two nuclei are shown. The three dark spots in the nuclei indicate a gain for this region of chromosome 7. C. Left panel, phase contrast microscopy of PriGO8A cells grown in the presence of laminin; right panel, phase contrast microscopy of PriGO8A cells grown in the absence of laminin, showing neurosphere formation. D. Immunofluorescence microscopy for nestin in PriGO8A cells growing on laminin. Cells were counterstained with DAPI. A control in which the primary antibody was omitted during staining is also shown. percentage of cells expressing TUJ1 and the percentage of cells expressing GFAP, indicating that PriGO8A cells differentiate along both neuronal and astrocytic lineages in the presence of serum. Although double labeling experiments were not performed here, the percentages of positive cells suggest that some of the differentiation observed is aberrant, giving rise to TUJ1/GFAP double positive cells, as observed previously $[33,32]$.

\section{Effects of PTEN, PKCı and Lgl1 on GTIC differentiation}

To assess the effects of PTEN on differentiation, PriGO8A cells were engineered for inducible expression of PTEN as described above for U87MG cells. Treatment with doxycycline induced PTEN expression in a dosedependent fashion (Figure 6A). As in U87MG cells, PTEN induction reduced the phosphorylation of transduced Lg11 (Figure 6B). PTEN-induction in PriGO8A cells significantly increased the relative proportion of cells that were TUJ1 positive (Figure 6C and D). This was not seen in unmodified PriGO8A cells treated with doxycycline, showing that the effect is dependent on PTEN induction (Figure 6D). PTEN induction had no effect on differentiation along the astrocytic lineage (Figure 6D).

To assess the role of $\mathrm{PKCl}$ in PriGO8A differentiation, transient knockdown of $\mathrm{PKCl}$ was performed using two different RNA duplexes at a 10 $\mathrm{nM}$ concentration (Figure 7A). Knockdown with both duplexes, but not with a control duplex used at the same concentration, resulted in increased differentiation along the neuronal lineage without any effects on astrocytic differentiation (Figure 7B and C).

To assess the role of Lgl1 phosphorylation, PriGO8A cells were transduced with lentiviral vector expressing Lgl3SA. As with U87MG cells, Lgl3SA, but not Lgl1, showed marked membrane localization in mitotic cells (Figure 8A). As with PTEN transduction and $\mathrm{PKC}$ knockdown, transduction of Lgl3SA also induced PriGO8A cell differentiation along the neuronal lineage, but not the astrocytic lineage (Figure $8 \mathrm{~B}$ and $8 \mathrm{C}$ ).

GTICs from different patients have been reported to show variability in their differentiation behavior [5]. To determine the generalizability of the above findings, the effects of $\mathrm{PKC} \mathrm{C}$ knockdown and Lgl3SA expression on GTIC differentiation were also assessed in cell populations isolated from two other patients, designated PriGO9A and PriGO7A. As with PriGO8A cells, PriGO9A and PriGO7A cells were able to form neurospheres when grown in the absence of laminin, were uniformly nestin positive and were able to undergo differentiation along neuronal and astrocytic lineages when exposed to serum (Figure 9A-B and 10A-B). Western blot analysis of PriGO9A and PriGO7A cells for expression of PTEN, Lgl1 and PKCı was shown in Figure 4A. Both show similar levels of 
$\mathrm{PKC} 1$ and $\mathrm{Lgl1}$ to those seen in PriGO8A cells. PriGO9A cells showed very low but detectable levels of PTEN, while PriGO7A cells show higher levels. PriGO7A cells were also examined by chromogenic in situ hybridization for $E G F R$ copy number, which showed amplification in this region (Figure 10A). Both knockdown of $\mathrm{PKCl}$ and Lg13SA transduction induced differentiation along the neuronal lineage in PriGO9A and PriGO7A cells without affecting astrocytic differentiation, similar to what was observed in PriGO8A cells (Figures 9C and 10C).

\section{DISCUSSION}

Partial or complete loss of the tumor suppressor PTEN, by either hemizygous deletion, homozygous deletion or mutation, is a frequent event in glioblastoma. In this study, we demonstrate that loss of this tumor suppressor leads to the inactivation of a second protein with tumor suppressor-like functions, Lgl1. Previous work has shown both that Lgl is inactivated by phosphorylation and that the atypical PKCs are responsible for this [20,22]. The key finding here is the link between this and the loss
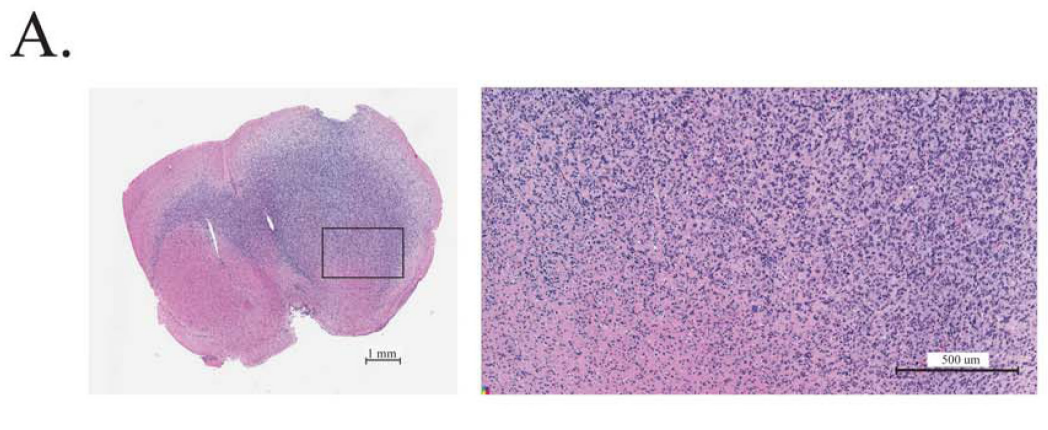

B.
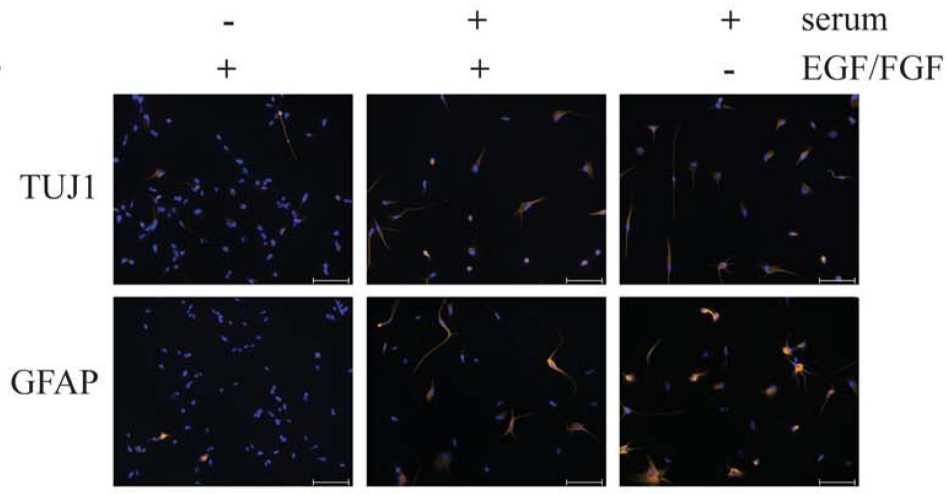

C.

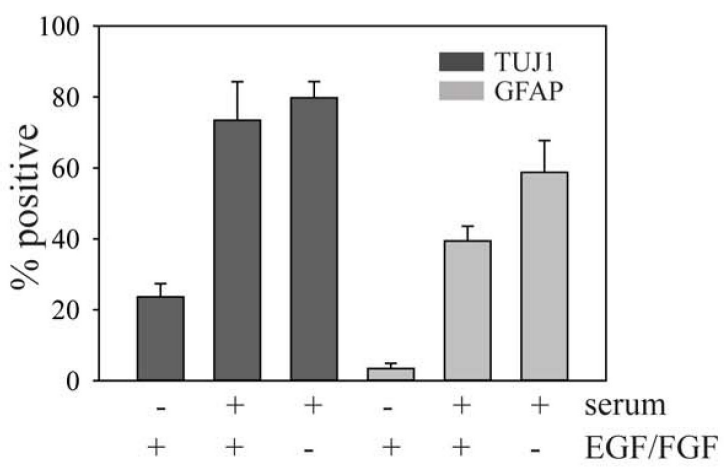

Figure 5: In vivo growth of PriGO8A cells and differentiation of PriGO8A cells in response to serum and/or growth factor withdrawal. A. Growth of PriGO8A cells three months after intrastriatal injection in the right hemisphere. Hematoxylin and eosin stained whole brain sections (left panel) show extensive growth and invasion into the left hemisphere across the corpus callosum. The close-up (right panel) shows a border area between cancer cells and normal brain. B.PriGO8A cells were grown in: regular media; media supplemented with $10 \%$ fetal calf serum; media supplemented with $10 \%$ fetal calf serum without EGF and FGF growth factors. Seven days later cells were fixed and immunofluorescence microscopy was performed for either TUJ1 or GFAP. C. Quantitation of the results in B. Data are shown as the mean \pm SE. 
of PTEN: we show that restoration of PTEN results in a decrease in Lgl1 phosphorylation as well as an increase in its membrane association, which is a marker of functional Lg11. The effects of PTEN on Lgl1 phosphorylation were seen both in U87MG cells and in GTICs. This finding represents a second mechanism by which Lgll can be inactivated in cancer cells, as previous studies have shown that Lg11 is downregulated at both the mRNA and protein levels in several cancer types, including colorectal cancer and melanoma, compared to normal tissue [24,25]. Lgl1 inactivation therefore appears to be a very frequent event in human cancers.

The effects of the PTEN/aPKC/Lgl1 pathway on the differentiation state of glioblastoma tumor initiating cells (GTICs) were assessed. GTICs were isolated from multiple patients using previously described culture techniques that preserve their ability to generate tumors in mice that mimic the pathology of human glioblastoma [32,5]. Cultures were screened for PTEN expression levels. GTICs from one patient (PriGO8A cells) were chosen for detailed study, as these showed complete loss of PTEN expression, in common with U87MG cells. PriGO8A cells
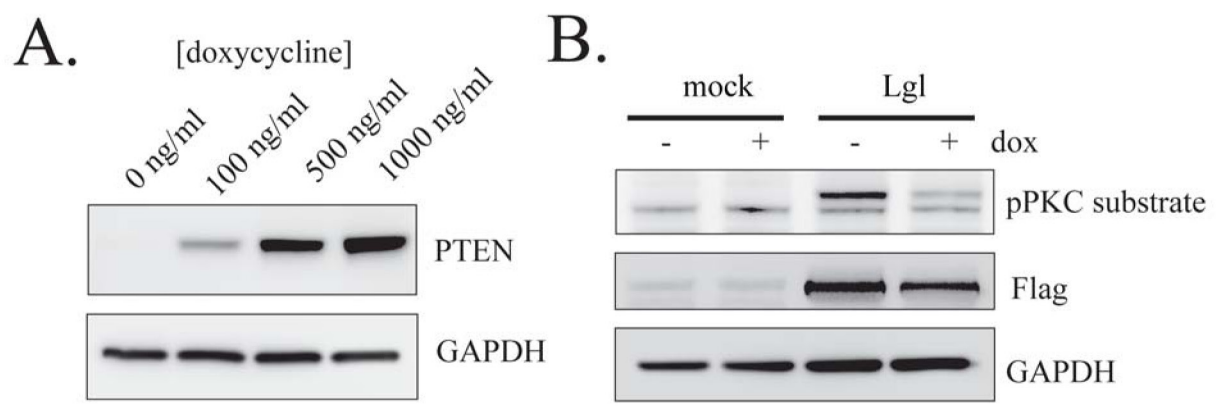

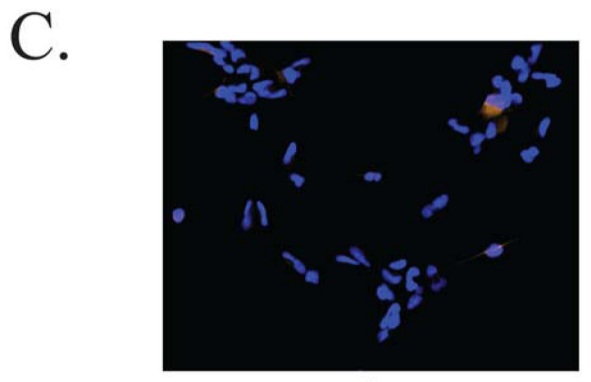

no dox
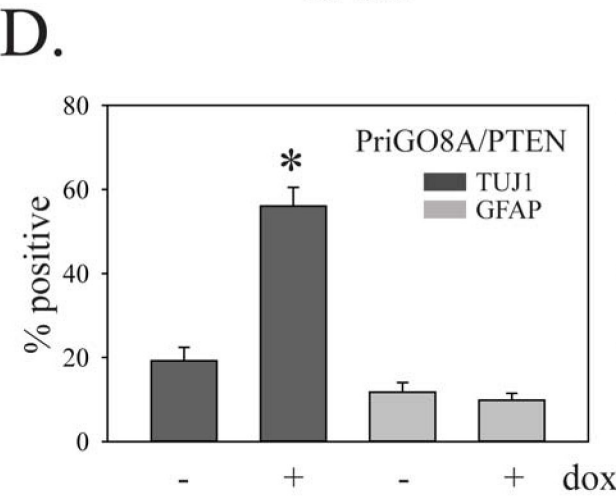

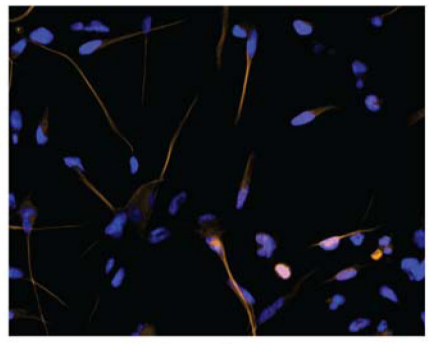

plus dox

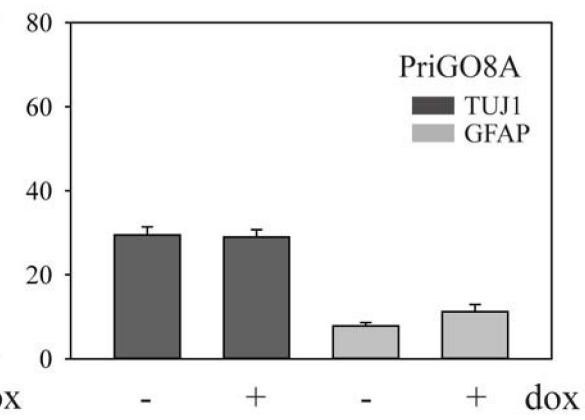

Figure 6: Effects of PTEN on PriGO8A differentiation. A. PriGO8A cells were transduced with Tet activator lentivirus and selected with G418 for four days. Selected cells were then transduced with inducible PTEN lentivirus and selected with puromycin for four days. Selected cells were treated with the indicated concentrations of doxycycline for $48 \mathrm{~h}$. Total cell lysates were then analyzed by Western blotting for expression of PTEN. B. PriGO8A cells with inducible PTEN were treated with $500 \mathrm{ng} / \mathrm{ml}$ doxycycline for two days and then either mock transduced or transduced with lentivirus constitutively expressing wild-type Lgl1. One day later cells were switched to media without EGF. Total cell extracts were collected one day later and analyzed by Western blotting with antibodies to phospho-PKC substrate and Flag epitope. C. PriGO8A cells with inducible PTEN were untreated or treated with $500 \mathrm{ng} / \mathrm{ml}$ doxycycline for 7 days. Cells were then fixed and immunocytochemistry for TUJ1 and GFAP expression was performed. D. Quantitation of data from C. The left graph shows doxycycline treatment of PriGO8A cells with inducible PTEN. The right graph shows doxycycline treatment of parental PriGO8A cells (as a control for effects of doxycycline alone). Quantitation was performed as described in Material and Methods. Data are shown as the mean \pm SE. * indicates a $\mathrm{p}$ value less than 0.05 . 
showed the common features of GTICs that have been described previously in the literature [33,5], including nestin expression, the ability to form neurospheres, the
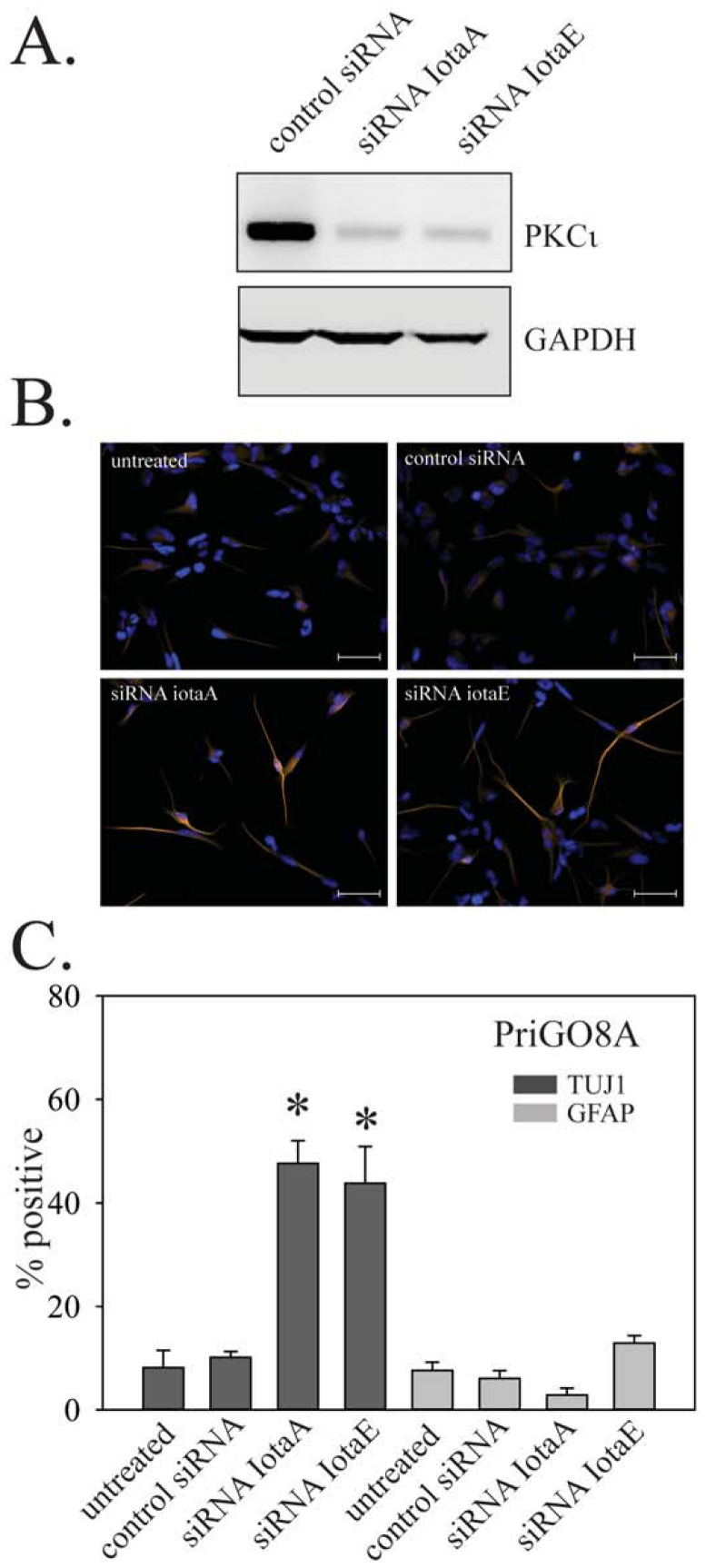

Figure 7: Effects of PKCı on PriGO8A differentiation. A. PriGO8A cells were transfected with control RNA duplex or two different RNA duplexes targeting PKCl. Three days later, total cell lysates were collected and analyzed for $\mathrm{PKCl}$ expression by Western blotting. B. Cells were transfected as in A. Seven days later cells were fixed and immunofluorescence with antibodies to TUJ1 and GFAP was performed. Representative images of TUJ1 immunofluorescence are shown. C. Quantitation of TUJ1 and GFAP immunofluorescence seven days after $\mathrm{PKCl}$ knockdown. Quantitation was performed as described in Material and Methods. Data are shown as the mean \pm SE. * indicates a $\mathrm{p}$ value less than 0.05 . ability to differentiate along both neuronal and astrocytic lineages in response to serum exposure, and the ability to form invasive tumors in immunocompromised mice.

PriGO8A cells were engineered for inducible expression of PTEN. As in U87MG cells, induction of PTEN in PriGO8A cells reduced the phosphorylation of Lgl1. Induction of PTEN also resulted in differentiation. This finding is similar to previous findings in adult neural stem cells and other non-cancer stem cell types, where

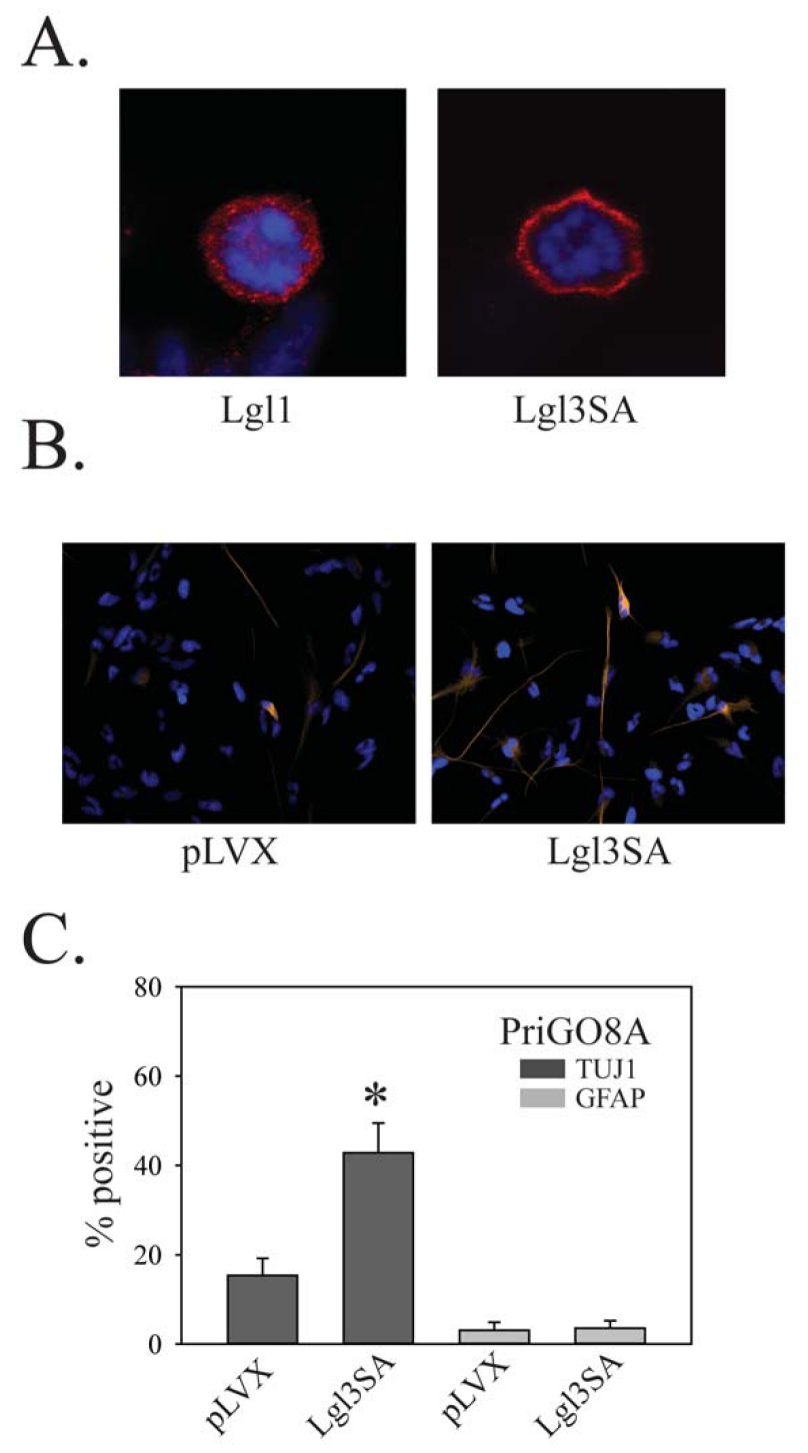

Figure 8: Effects of Lgl3SA on PriGO8A differentiation. A. PriGO8A cells were transduced with either Lgl1 (left) or Lgl3SA (right). Four days later cells were fixed and immunofluorescence with anti-Flag antibody was performed. Representative examples of mitotic cells are shown. B. PriGO8A cells were transduced with either empty vector control (pLVX) or vector expressing Lgl3SA. Seven days later cells were fixed and immunofluorescence for TUJ1 and GFAP was performed. Representative images for TUJ1 staining are shown. C. Quantitation of TUJ1 and GFAP immunofluorescence seven days after transduction with Lg13SA. Data are shown as the mean $\pm \mathrm{SE}$. * indicates a $\mathrm{p}$ value less than 0.05 . 
loss of PTEN represses differentiation [34]. Although serum exposure resulted in differentiation of these cells along both neuronal and astrocytic lineages, with PTEN induction differentiation was exclusively along the neuronal lineage. This contrasts with the previously reported effects of BMP-4 on GTIC differentiation, where differentiation occurred primarily along the astrocytic lineage [35]. In most human glioblastoma tumors, the bulk of the cells are GFAP-positive, indicating that differentiation occurs primarily along the astrocytic lineage. Results here suggest the possibility that this is a consequence of strong repression of neuronal differentiation by PTEN loss and subsequent PI 3-kinase pathway activation.

As with PTEN induction, knockdown of $\mathrm{PKCl}$ also reduced the phosphorylation of Lgl1. In addition, knockdown of $\mathrm{PKCl}$ in PriGO8A cells resulted in differentiation along the neuronal lineage, phenocopying what was seen with PTEN induction. This indicates that $\mathrm{PKCl}$ is the key mediator of PI 3-kinase pathway effects on GTIC differentiation.

A non-phosphorylatable constitutively-active mutant of Lgl1 (designated Lg13SA) was also expressed in PriGO8A cells. This was able to associate with the cell membrane; importantly this indicates that the human homologs of the Drosophila tumor suppressors Scribble
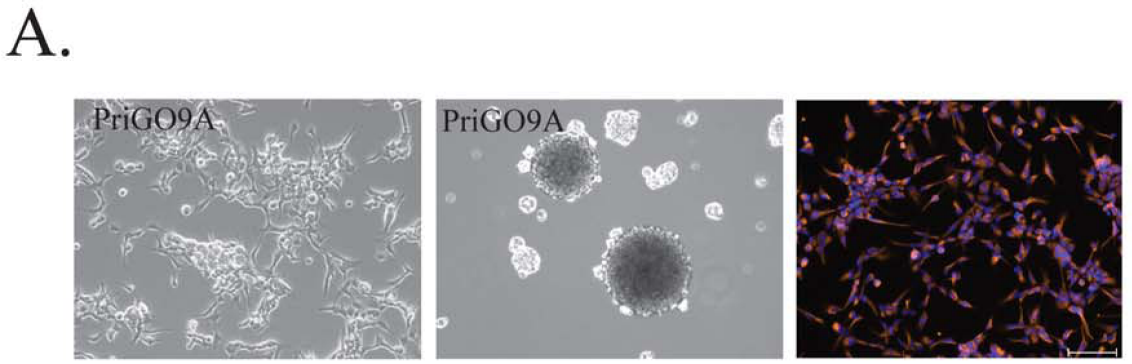

B.
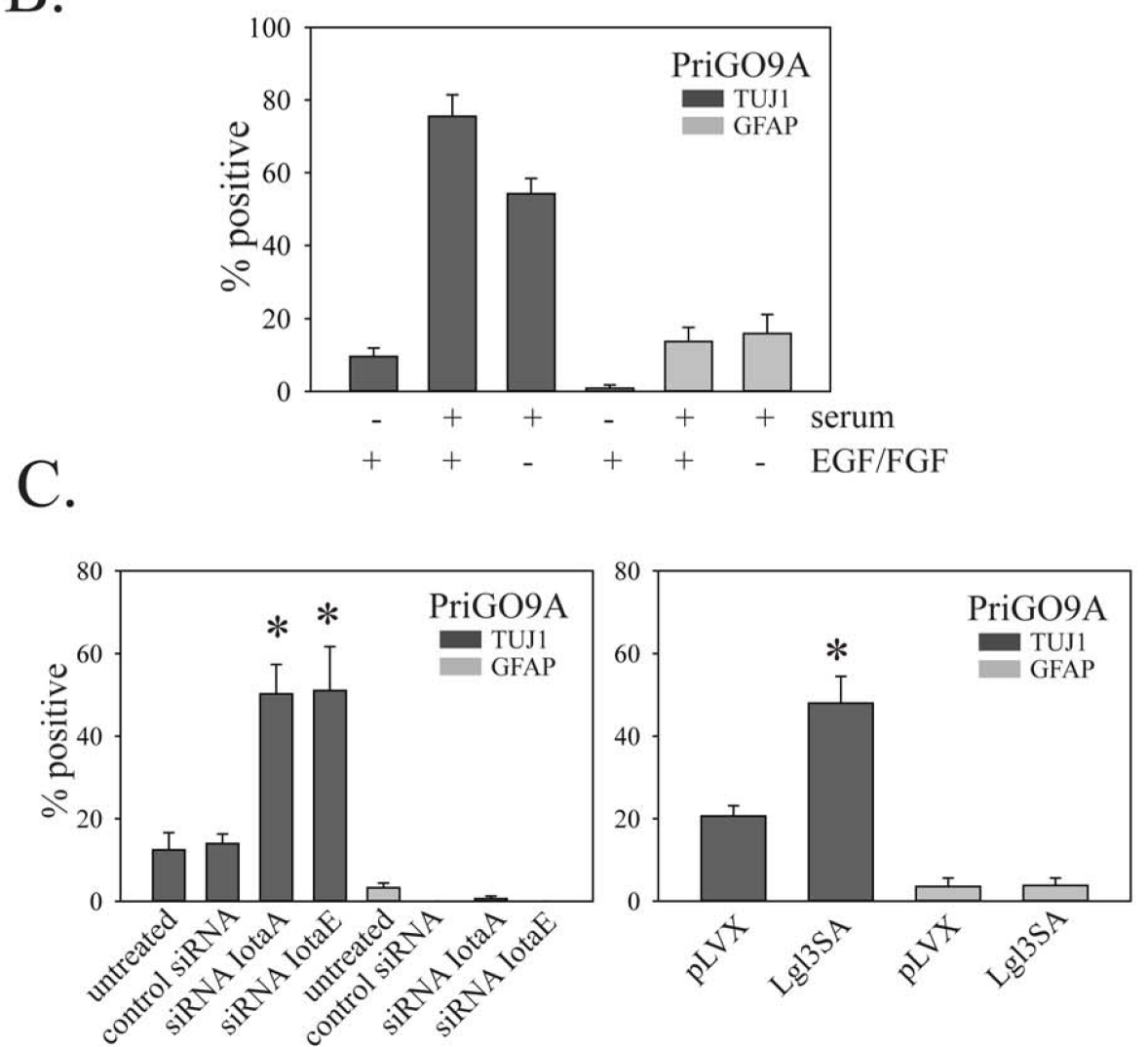

Figure 9: Effects of PKC and Lgl3SA on PriGO9A cell differentiation. A. Morphology under phase contrast microscopy (top left panel), neurosphere formation (top middle panel) and nestin immunofluorescence (top right panel) for PriGO9A cell cultures. B. Differentiation of PriGO9A cells after exposure to serum with or without growth factor withdrawal, determined as described in Figure 5. C. Bar graphs show the effects of $\mathrm{PKC}$ knockdown (left panel) and Lgl3SA transduction (right panel) on PriGO9A differentiation, determined as described in Figure 7. 
and Discs large are functional in these cells, as these proteins are known to be required for $\mathrm{Lgl}$ membrane association [16]. As with PTEN induction and $\mathrm{PKC1}$ knockdown, expression of Lg13SA induced differentiation exclusively along the neuronal lineage, indicating that $\mathrm{Lgl1}$ is a key substrate mediating the effects of $\mathrm{PKCl}$ on GTIC differentiation.

The effects of Lgl3SA were also assessed in GTIC

A.
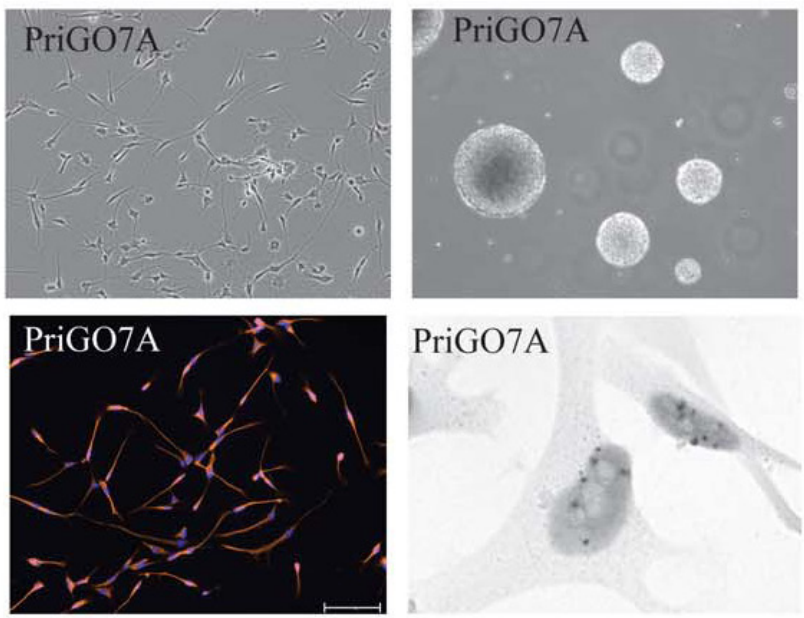

PriGO7A

B.
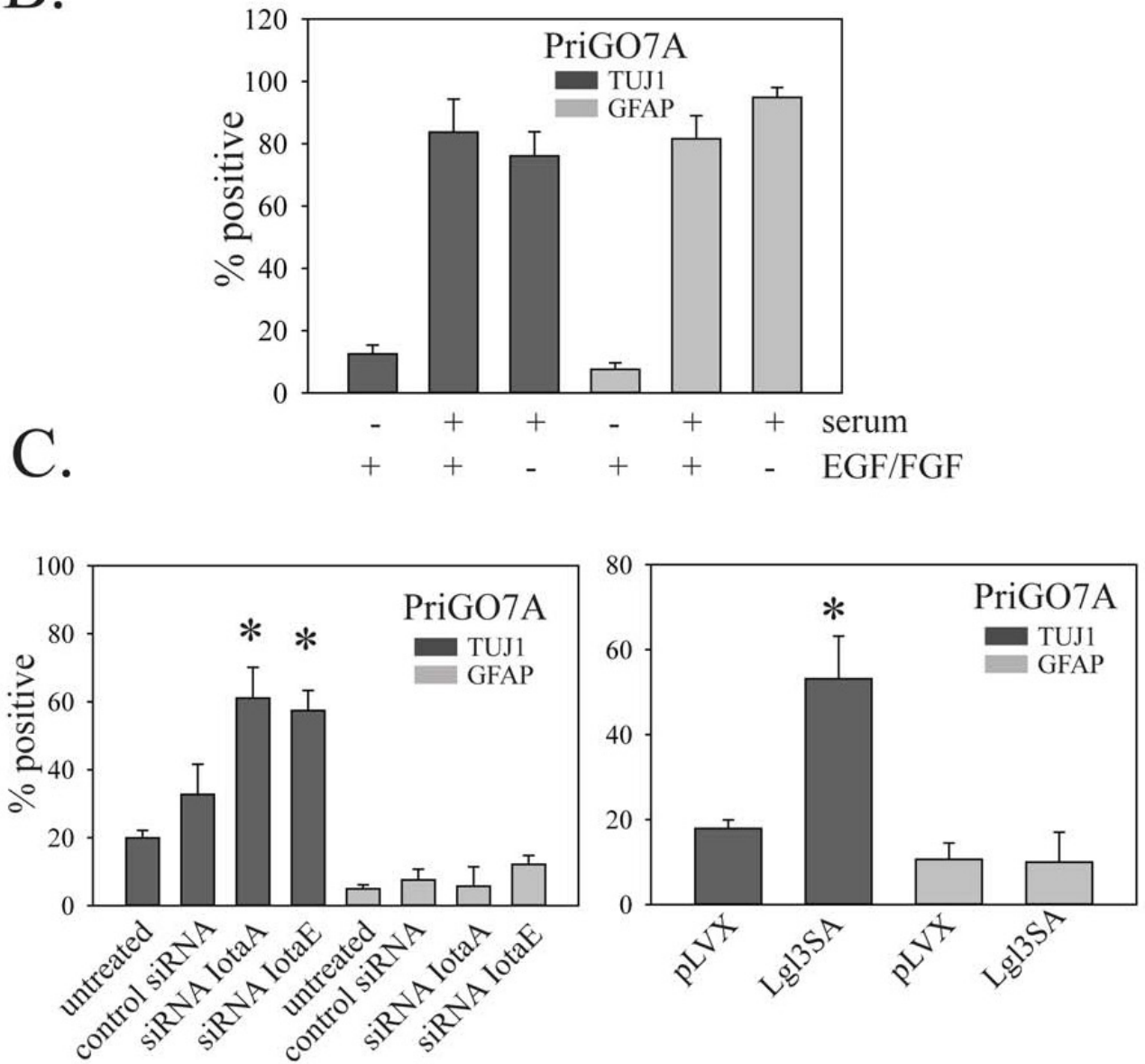

Figure 10: Effects of PKC and Lgl1 on PriG07A differentiation. Morphology under phase contrast microscopy (top left panel), neurosphere formation (top right panel), nestin immunofluorescence (lower left panel) and EGFR chromogenic in situ hybridization for PriGO7A cells (lower right panel). B. Differentiation of PriGO7A cells after exposure to serum or growth factor withdrawal. C. Bar graphs show the effects of $\mathrm{PKC}$ knockdown (left panel) and Lgl3SA transduction (right panel) on PriGO7A differentiation. 
cells from two additional patients. PriGO9A cells, which showed low but detectable levels of PTEN, also underwent differentiation along a neuronal lineage when transduced with Lg13SA. This effect was also seen in PriGO7A cells. These cells express high levels of PTEN, but show evidence of amplification of EGFR. Thus the role for Lgl1 does not appear to be restricted to GTICs in which PTEN expression is completely lost, but is also functional in GTICs where the PI 3-kinase pathway is hyperactivated by other mechanisms such as EGFR amplification.

The above findings for $\mathrm{PKCl}$ and $\mathrm{Lgll}$ are consistent with previous studies in Drosophila showing that atypical PKC and Lgl mediate the decision between neuroblast self-renewal and differentiation [17]. The data here show that this pathway is conserved in human GTICs and, critically, is linked to PTEN and PI 3-kinase signaling pathway status. The fact that partial or complete loss of PTEN expression is very common suggests that it is an early event in gliomagenesis. Thus inactivation of Lgl1 may also be an early event in gliomagenesis, leading to the expansion of a population of undifferentiated tumor initiating cells, the cell population that is the key driver for glioblastoma malignancy [2].

\section{MATERIALS AND METHODS}

\section{Antibodies and chemicals:}

Anti-Flag M2 mouse monoclonal antibody, nonmuscle myosin II rabbit polyclonal antibody and GFAP mouse monoclonal were from Sigma-Aldrich (Oakville, ON, Canada). PKCl mouse monoclonal and phosphothreonine $555 \mathrm{PKCl}$ rabbit polyclonal antibodies were from BD Transduction Laboratories (Mississauga, ON, Canada) and Invitrogen (Carlsbad, CA, USA) respectively. The Akt goat polyclonal was from Santa Cruz Biotechnology (Santa Cruz, CA, USA). PhosphoAKT (Ser473) rabbit polyclonal, phospho-(Ser) PKC Substrate (P-S3-101) antibody (a mix of three rabbit monoclonal antibodies), and PTEN rabbit monoclonal antibody were from Cell Signaling Technology (Danvers, MA, USA). Lgl1 rabbit polyclonal antibody and GAPDH mouse monoclonal (6C5) antibody were from Abcam (Cambridge, MA, USA). TUJ1 rabbit monoclonal antibody was from Covance (Princeton, NJ, USA). Nestin mouse monoclonal antibody was from R\&D Systems (Minneapolis, MN, USA).

\section{Cell Culture:}

U87MG cells were grown in Dulbecco's Modified Eagle medium supplemented with 100 units $/ \mathrm{ml}$ penicillin, $100 \mu \mathrm{g} / \mathrm{ml}$ streptomycin and $10 \%$ fetal bovine serum at $37^{\circ} \mathrm{C}$ and $5 \% \mathrm{CO}_{2}$. Cells were used at low passage number and were routinely checked and shown to be free of mycoplasma. GTIC cultures were isolated following a protocol approved by the Ottawa Hospital Research Ethics Board. Surgical samples were harvested from consented patients undergoing surgery for suspected glioblastoma (and without a history of previous lower grade brain tumor) using a Nico Myriad surgical device (NICO Corporation, Indianapolis, IN, USA). Cultures were digested with Accutase, filtered through $100 \mu \mathrm{M}$ and 40 $\mu \mathrm{M}$ nylon mesh filters, and plated on laminin-coated plates as described by Pollard et al. [5]. Accutase and laminin were from Sigma-Aldrich, Oakville, ON, Canada. Cultures were grown in Neurobasal A medium supplemented with B27, N2 (all from Life Technologies, Burlington, ON, Canada), EGF and FGF (Peprotech, Rocky Hill, NJ, USA) at $37^{\circ} \mathrm{C}$ in $5 \% \mathrm{O}_{2} / \mathrm{CO}_{2}$.

\section{Plasmid constructs:}

Full-length cDNA encoding Lgll mRNA was produced as described previously [14]. Site-directed mutagenesis was used to add an amino terminal Flag tag using the QuikChange XL Site-Directed Mutagenesis kit (Stratagene, La Jolla, CA, USA). Site-directed mutagenesis was also used to change the codons for serine 656,660 and 664 to alanine to generate Flagtagged Lg13SA cDNA. For constitutive lentiviral vector production, Flag-tagged wild-type Lgl or Lg13SA were PCR amplified to add Xbal and Sal1 5' and 3' restriction sites and subcloned into pLenti-CMV GFP Puro (Addgene Plasmid 17448) which had been digested with the same restriction enzymes to remove the GFP cDNA. For inducible expression, PTEN cDNA, Flag-tagged Lgl cDNA and Flag-tagged Lgl3SA cDNA were subcloned into the Tet-inducible lentiviral vector $\mathrm{pLVX}$-Tight-Puro (Clontech, Mountain View, CA, USA).

\section{Transduction with lentiviral vectors:}

Replication-incompetent lentiviral particles were made by the four-plasmid transfection method described by Wiederschain et al. [36]. U87MG were transduced by incubation for $24 \mathrm{~h}$ with lentivirus-containing supernatant added to regular media supplemented with $10 \mu \mathrm{g} / \mathrm{ml}$ polybrene (for U87MG transductions; polybrene was omitted for tumor initiating cell transductions). For inducible expression, cells were first transduced with lentivirus made with Tet-activator plasmid (Clontech, Mountain View, CA, USA) and selected with G418 $(500 \mu \mathrm{g} / \mathrm{ml})$; these cells were then transduced again with lentivirus made with the inducible cDNA vectors described above and selected with puromycin $(1 \mu \mathrm{g} / \mathrm{ml}$ for U87MG and $0.5 \mu \mathrm{g} / \mathrm{ml}$ for PriGO cells). 


\section{Western blotting:}

Western blotting was done as described previously [15]. Chemiluminescence from HRP conjugated secondary antibodies was detected with the Alpha Innotech Fluorchem system (Santa Clara, CA, USA) and quantitated using Alphaview software.

\section{Immunofluorescence microscopy:}

Immunofluorescence microscopy was done as described previously [14]. For differentiation experiments using TUJ1 and GFAP antibodies, images were taken of five random fields of view (chosen under DAPI filter) per condition per experiment. Analysis was carried out in ImageJ software (National Institutes of Health, Bethesda, Maryland, USA). The proportion of positive cells to total nuclei was assessed per condition $+/$ - SE for each experiment.

\section{Chromogenic in situ hybridization:}

Chromogenic in situ hybridization was used to detect EGFR gene copy number changes in PRIGO cells. ZytoDot SPEC EGFR Probe (Cat. ZTV-C-3007-400) and ZytoDot CISH Implementation Kit were from Cedarlane (Burlington, ON, Canada) and were used according to the manufacturer's recommendations.

\section{Intracerebral xenografts:}

Experiments were carried out in accordance with the recommendations of the Animal Care Committee at the University of Ottawa.

$5 \times 10^{5}$ PriGO cells in $10 \mu \mathrm{L}$ sterile PBS were injected intrastriatal (right side of the skull approximately $0.5 \mathrm{~mm}$ above the coronal suture and $2 \mathrm{~mm}$ from the sagittal suture) into 4-6 week old SCID/beige mice (Charles River Laboratories, Wilmington. MA). Endpoint was reached after approximately three months when mice became symptomatic. Whole brains were harvested, formalin fixed and paraffin embedded. Sections were cut, stained with hematoxylin and eosin, and reviewed by a neuropathologist (JW).

\section{Statistics:}

SigmaPlot12 software was used for statistical analyses. Comparisons between two groups were performed using two-tailed t-tests with a $\mathrm{p}$ value $<0.05$ considered significant.

\section{ACKNOWLEDGEMENTS}

We thank Julie Wells and Sharon Kelly at the Ottawa Hospital Regional Cancer Centre for their invaluable help in obtaining patient consent for isolation of GTICs. This work was supported by a grant from the Canadian Institutes of Health Research (to IAJL). AG is supported by a Queen Elizabeth II Graduate Scholarship in Science and Technology.

\section{REFERENCES}

1. Stupp R, Mason WP, van den Bent MJ, Weller M, Fisher B, Taphoorn MJ, Belanger K, Brandes AA, Marosi C, Bogdahn U, Curschmann J, Janzer RC, Ludwin SK, Gorlia T, Allgeier A, Lacombe D, et al. Radiotherapy Plus Concomitant and Adjuvant Temozolomide for Glioblastoma. N Engl J Med. 2005; 352:987-996.

2. Singh SK, Hawkins C, Clarke ID, Squire JA, Bayani J, Hide T, Henkelman RM, Cusimano MD, Dirks PB. Identification of Human Brain Tumour Initiating Cells. Nature. 2004; 432:396-401.

3. Venere M, Fine HA, Dirks PB, Rich JN. Cancer Stem Cells in Gliomas: Identifying and Understanding the Apex Cell in Cancer's Hierarchy. Glia. 2011; 59:1148-1154.

4. Munoz DM and Guha A. Mouse Models to Interrogate the Implications of the Differentiation Status in the Ontogeny of Gliomas. Oncotarget. 2011; 2:590-598.

5. Pollard SM, Yoshikawa K, Clarke ID, Danovi D, Stricker S, Russell R, Bayani J, Head R, Lee M, Bernstein M, Squire JA, Smith A, Dirks P. Glioma Stem Cell Lines Expanded in Adherent Culture Have Tumor-Specific Phenotypes and Are Suitable for Chemical and Genetic Screens. Cell Stem Cell. 2009; 4:568-580.

6. Bao S, Wu Q, McLendon RE, Hao Y, Shi Q, Hjelmeland AB, Dewhirst MW, Bigner DD, Rich JN. Glioma Stem Cells Promote Radioresistance by Preferential Activation of the DNA Damage Response. Nature. 2006; 444:756-760.

7. Chen J, Li Y, Yu TS, McKay RM, Burns DK, Kernie SG, Parada LF. A Restricted Cell Population Propagates Glioblastoma Growth After Chemotherapy. Nature. 2012; 488:522-526.

8. Verhaak RG, Hoadley KA, Purdom E, Wang V, Qi Y, Wilkerson MD, Miller CR, Ding L, Golub T, Mesirov JP, Alexe G, Lawrence M, O'Kelly M, Tamayo P, Weir BA, Gabriel S, et al. Integrated Genomic Analysis Identifies Clinically Relevant Subtypes of Glioblastoma Characterized by Abnormalities in PDGFRA, IDH1, EGFR, and NF1. Cancer Cell. 2010; 17:98-110.

9. Weber GL, Parat MO, Binder ZA, Gallia GL, Riggins GJ. Abrogation of PIK3CA or PIK3R1 Reduces Proliferation, Migration, and Invasion in Glioblastoma Multiforme Cells. Oncotarget. 2011; 2:833-849.

10. Vanhaesebroeck B and Alessi DR. The PI3K-PDK1 
Connection: More Than Just a Road to PKB. Biochem J. 2000; 346 Pt 3:561-576.

11. Le Good JA, Ziegler WH, Parekh DB, Alessi DR, Cohen P, Parker PJ. Protein Kinase C Isotypes Controlled by Phosphoinositide 3-Kinase Through the Protein Kinase PDK1. Science. 1998; 281:2042-2045.

12. Fields AP and Regala RP. Protein Kinase C Iota: Human Oncogene, Prognostic Marker and Therapeutic Target. Pharmacol Res. 2007; 55:487-497.

13. Baldwin RM, Parolin DA, Lorimer IA. Regulation of Glioblastoma Cell Invasion by PKC Iota and RhoB. Oncogene. 2008; 27:3587-3595.

14. Baldwin RM, Barrett GM, Parolin DA, Gillies JK, Paget JA, Lavictoire SJ, Gray DA, Lorimer IA. Coordination of Glioblastoma Cell Motility by PKCiota. Mol Cancer. 2010; 9:233-246.

15. Paget JA, Restall IJ, Daneshmand M, Mersereau JA, Simard MA, Parolin DA, Lavictoire SJ, Amin MS, Islam S, Lorimer IA. Repression of Cancer Cell Senescence by PKCiota. Oncogene. 2012; 31:3584-3596.

16. Humbert P, Russell S, Richardson H. Dlg, Scribble and Lgl in Cell Polarity, Cell Proliferation and Cancer. Bioessays. 2003; 25:542-553.

17. Lee CY, Robinson KJ, Doe CQ. Lgl, Pins and APKC Regulate Neuroblast Self-Renewal Versus Differentiation. Nature. 2006; 439:594-598.

18. Klezovitch O, Fernandez TE, Tapscott SJ, Vasioukhin V. Loss of Cell Polarity Causes Severe Brain Dysplasia in Lg11 Knockout Mice. Genes Dev. 2004; 18:559-571.

19. Knoblich JA. Asymmetric Cell Division: Recent Developments and Their Implications for Tumour Biology. Nat Rev Mol Cell Biol. 2010; 11:849-860.

20. Plant PJ, Fawcett JP, Lin DC, Holdorf AD, Binns K, Kulkarni S, Pawson T. A Polarity Complex of MPar-6 and Atypical PKC Binds, Phosphorylates and Regulates Mammalian Lgl. Nat Cell Biol. 2003; 5:301-308.

21. Dahan I, Yearim A, Touboul Y, Ravid S. The Tumor Suppressor Lg11 Regulates NMII-A Cellular Distribution and Focal Adhesion Morphology to Optimize Cell Migration. Mol Biol Cell. 2012; 23:591-601.

22. Betschinger J, Eisenhaber F, Knoblich JA. PhosphorylationInduced Autoinhibition Regulates the Cytoskeletal Protein Lethal (2) Giant Larvae. Curr Biol. 2005; 15:276-282.

23. Grifoni D, Garoia F, Schimanski CC, Schmitz G, Laurenti E, Galle PR, Pession A, Cavicchi S, Strand D. The Human Protein Hugl-1 Substitutes for Drosophila Lethal Giant Larvae Tumour Suppressor Function in Vivo. Oncogene. 2004; 23:8688-8694.

24. Schimanski CC, Schmitz G, Kashyap A, Bosserhoff AK, Bataille F, Schafer SC, Lehr HA, Berger MR, Galle PR, Strand S, Strand D. Reduced Expression of Hugl-1, the Human Homologue of Drosophila Tumour Suppressor Gene Lgl, Contributes to Progression of Colorectal Cancer. Oncogene. 2005; 24:3100-3109.
25. Kuphal S, Wallner S, Schimanski CC, Bataille F, Hofer P, Strand S, Strand D, Bosserhoff AK. Expression of Hugl-1 Is Strongly Reduced in Malignant Melanoma. Oncogene. 2006; 25:103-110.

26. Kashyap A, Zimmerman T, Ergul N, Bosserhoff A, Hartman U, Alla V, Bataille F, Galle PR, Strand S, Strand D. The Human Lgl Polarity Gene, Hugl-2, Induces MET and Suppresses Snail Tumorigenesis. Oncogene. 2012, 32:1396-1407.

27. Yamanaka T, Horikoshi Y, Sugiyama Y, Ishiyama C, Suzuki A, Hirose T, Iwamatsu A, Shinohara A, Ohno S. Mammalian Lgl Forms a Protein Complex With PAR-6 and APKC Independently of PAR-3 to Regulate Epithelial Cell Polarity. Curr Biol. 2003; 13:734-743.

28. Ishii N, Maier D, Merlo A, Tada M, Sawamura Y, Diserens AC, Van Meir EG. Frequent Co-Alterations of TP53, P16/ CDKN2A, P14ARF, PTEN Tumor Suppressor Genes in Human Glioma Cell Lines. Brain Pathol. 1999; 9:469-479.

29. Rafalski VA and Brunet A. Energy Metabolism in Adult Neural Stem Cell Fate. Prog Neurobiol. 2011; 93:182-203.

30. Leontieva OV, Natarajan V, Demidenko ZN, Burdelya LG, Gudkov AV, Blagosklonny MV. Hypoxia Suppresses Conversion From Proliferative Arrest to Cellular Senescence. Proc Natl Acad Sci U S A. 2012; 109:1331413318.

31. Higgins DM, Wang R, Milligan B, Schroeder M, Carlson B, Pokorny J, Cheshier SH, Meyer FB, Weissman IL, Sarkaria JN, Henley JR. Brain Tumor Stem Cell Multipotency Correlates With Nanog Expression and Extent of Passaging in Human Glioblastoma Xenografts. Oncotarget. 2013; 4:792-801.

32. Fael Al-Mayhani TM, Ball SL, Zhao JW, Fawcett J, Ichimura K, Collins PV, Watts C. An Efficient Method for Derivation and Propagation of Glioblastoma Cell Lines That Conserves the Molecular Profile of Their Original Tumours. J Neurosci Methods. 2009; 176:192-199.

33. Lee J, Kotliarova S, Kotliarov Y, Li A, Su Q, Donin NM, Pastorino S, Purow BW, Christopher N, Zhang W, Park JK, Fine HA. Tumor Stem Cells Derived From Glioblastomas Cultured in BFGF and EGF More Closely Mirror the Phenotype and Genotype of Primary Tumors Than Do Serum-Cultured Cell Lines. Cancer Cell. 2006; 9:391-403.

34. Hill R and Wu H. PTEN, Stem Cells, and Cancer Stem Cells. J Biol Chem. 2009; 284:11755-11759.

35. Piccirillo SG, Reynolds BA, Zanetti N, Lamorte G, Binda E, Broggi G, Brem H, Olivi A, DiMeco F, Vescovi AL. Bone Morphogenetic Proteins Inhibit the Tumorigenic Potential of Human Brain Tumour-Initiating Cells. Nature. 2006; 444:761-765.

36. Wiederschain D, Wee S, Chen L, Loo A, Yang G, Huang A, Chen Y, Caponigro G, Yao YM, Lengauer C, Sellers WR, Benson JD. Single-Vector Inducible Lentiviral RNAi System for Oncology Target Validation. Cell Cycle. 2009; 8:498-504. 